\title{
Management of the Equipment Status in the Telecommunication Room Based on Sensors and RFID
}

\author{
Shan Yang ${ }^{1}$, Feng Guo ${ }^{1}$, Jinhui Zhao ${ }^{1}$, Yuan $\mathrm{Liu}^{2}$, Longjun Deng ${ }^{3, *}$ and Jinjun $\mathrm{Xu}^{3}$ \\ ${ }^{1}$ Information \& communication, State grid information \& communication Branch of Hubei Hubei, China \\ ${ }^{2}$ Information \& communication, State grid information \& communication Branch of Xianning Power Supply Company, Xianning, \\ China \\ ${ }^{3}$ Xiamen Great Power Geo Information Technology Co., Ltd., Xiamen, China, 361009. \\ *Corresponding author
}

\begin{abstract}
In order to realize the online monitor of the environment and energy consumption of the telecommunication room, we employed the environment sensor to monitor the temperature, humidity, and water leaching of the telecommunication room. Meanwhile, energy consumption sensors were utilized to monitor the consumption of the lighting, air conditioner, and power. Collected data were integrated to the resource management module with RFID tag. Afterwards, specific RFID was used to read the attached RFID tag to get the collected data and fed back to the certain machine or the presented operator. The practical demonstrated that our method could realize online monitoring. Based on the fast and reasonable distribution of the telecommunication resource, management of the field operation was improved. Furthermore, the management of the telecommunication was improved, and the profit was maximized.
\end{abstract}

Keywords-RFID; sensor; monitor; management

\section{INTRODUCTION}

With the fast development of the information technology, more and more equipment in the telecommunication room are utilized for the data transmission [1]. Meanwhile, the number of the corresponding assistant devices is increased. Along the manufacture and management of the power system [2], the information technology is one of the critical supports. Therefore, the telecommunication rooms play an important role for the stable operation of the power system. In case, any problem happened within the telecommunication room can be destructive to the management of the whole power system. It is imperative to guarantee the operation reliability of the telecommunication room [3].

In the past decades, the hybrid of the support system and monitoring apparatus for the computer room was developed for wide application. For example, Choi, Jeon and Kim (2007) [4] proposed a method to monitor the cooling performance of a hybrid refrigeration system for the telecommunication equipment rooms. They thought that the optimum control of the PCB surface temperature was very important to obtain high performance and operation reliability of telecommunication equipment. Thus, the cooling characteristics of telecommunication equipment were measured and analyzed as a function of the equipment's heat density. Furthermore, the performance of a novel hybrid refrigeration system for telecommunication equipment rooms was measured at various operating conditions. Chen (2012) [5] proposed a surveillance system for the computer room that was used for the maritime communications. Yang (2005) [6] proposed a system which consisted of the real surveillance module and management module for the computer room's security. Okundamiya, Emagbetere and Ogujor (2014) [7] proposed a type of green technology about the energy costs and carbon footprint of operating mobile telecommunication sites. They designed a system, which constrained the generation and distribution of power to reliably satisfy the energy demand, meanwhile, ensuring the safe operation of the system based on the genetic algorithm. Bu, Lu and Huang (2010) [8] introduced the structure of equipment monitoring system of network center computer room in campus and the function of subsystem. The system adopted the distributed building control room, TCP / IP network and RS- 485 /RS- 232 bus structure to combine the field controller with control module and compose system control network.

In order to guarantee the operation stability of the telecommunication room and the efficiency of the daily maintain, we proposed a system consisted of specific sensors, data module with RFID tags and RFID Reader. Via scanning the RFID tags, data are fed back to the certain person or machine for further analysis and management [9].

The paper is organized as follows: (1) section 1 introduces the background of our research. (2) Section 2 demonstrates the potential problems of the telecommunication room. (3) Sections 3 introduce the system based on the specific sensors and the RFID. (4) We provide the experiment results and conclusion.

\section{THE POTENTIAL PROBLEMS OF THE TELECOMMUNICATION ROOM}

\section{A. Fire and Smoke}

The fire and smoke can be mainly caused by the following points. 
(1) Interior decoration of the telecommunication room used large quantities of combustible materials, such as wood, plywood, plastic board and so on. Meanwhile, many materials for the heat insulation are utilized for the ventilation pipe. All the referred materials can potentially lead to reduction of the fire resistance of the telecommunication room [10].

(2) In the telecommunication room, a large number of electrical equipment is placed here. Improper and any error installation of the devices can cause the short circuit and heavy load, which can both lead to the fire accident.

(3) Improper management of the telecommunication room can cause fire accident. For example, the flammable and explosive goods are not treated in time or in the use of flammable cleaning agent to wipe the machine equipment and floor [11].

(4) In the condition of the improper ground handling of the communication equipment in the operation room, polyester, acrylic, polyvinyl chloride and other clothing or PVC slippers of the staff are prone to bring static electricity spark, which will cause the fire accident.

(5) Electronic devices need to work continuously for a long time. If a failure occurs, it is likely that the insulator is punctured. The high impedance element is exposed to the bad heat, and therefore the fire occurs.

Thus it is important to consider the problem of the early detection of the smoke, which is critical to control the fire accident.

\section{B. High Temperature and the Humidity}

Considering large quantities of the semi-semiconductors, resistors and capacitors used by telecommunication equipment, the high temperature can make the components work unstable, and even out of work. Furthermore, it leads the equipment failure. Therefore, it is necessary to control the temperature of the telecommunication room within a certain range.

Similarly for the humidity, the low humidity brings can cause static electricity, and high humidity can cause phenomenon of galvanic circle.

\section{Power Wasting}

In order to keep the comfortable environment for the operation of the equipment, all the air conditioner and light works in a fixed model. Meanwhile, the machine work as the information transmission is not utilized all the time. Thus, it is necessary to understand the energy consuming of the telecommunication room and final the optimal scheme of energy saving.

\section{The Other Problems}

There are some other problems, including exception and out of work of the air conditioner, illegal infiltration that is related to the data security, fire and leakage and so on. The problem about the power supply mainly includes the break down about the power supply system and the supply interruption. It can cause the mistakes of information transmitting, and interrupt the reliability operation of the devices.

\section{SYSTEM CONSISTING OF SENSORS AND RFID}

In order to solve the above problems, we proposed a system based on a wireless sensor network, which include environment sensors and energy cost sensors, and RFID [12]. The system provides a high density of sensors in a given area. The wireless system is composed of a sensor node and a base station. The sensor node consists of specific sensors, including temperature sensors, relative humidity sensors and so on.

\section{A. Temperature and Relative Humidity Sensors}

The components of the electronic devices are significant affected by the temperature and humidity. Monitoring and control of indoor climate conditions is thus crucial in keeping the stable state of equipment in the telecommunication room.

Wireless temperature and humidity sensor was employed in our system to monitor the temperature and the relative humidity. The temperature sensors provide a precise characterization of the fluctuations in microclimate environmental conditions. Continuous monitoring for a longer period of time will also be achieved as the sensor nodes will be configured to run in a low duty cycle operation, thus conserving battery life.

\section{B. Water Leakage Sensor}

Water leakage in the telecommunication room poses risk to the electronic devices and incurs great economic costs. In order to find the leaks with less labor force and on time, the water leakage sensor is utilized in our system to monitor whether the telecommunication room has dumping. Early detection can provide instant control action.

\section{Smoke Sensor}

In case a fire accident happed to the telecommunication room, it will lead to paralysis of the entire power system network, and cause huge loss and influence. Besides if the economics losses, and destruction of information, data and so on will bring unimaginable influence to the management of the power system. Therefore, it is important to ensure the security of the telecommunication room.

In our system, the wireless smoke sensor is employed to monitor the smokes of the telecommunication room, and make early alarm to the specified machine or people.

\section{Energy Monitor}

In order to realize the energy saving, we employed the intelligent power meter and battery collection module to monitor the electric supply and accumulators respectively. Furthermore, DC monitoring is used to record the data of the current of the switch on the DC screen. All the energy consuming data can be recorded for further analysis, than finally realize the application of energy saving

\section{E. Other Sensors}

In order to realize the green telecommunication, which is defined as maintain the stable work state with less labor force, some other sensors are employed in our system, such as the power supply sensor, Infrared lattice and temperature sensor. 


\section{F. RFID}

Radio frequency identification (RFID) [13] is a generic term that is used to describe a system that transmits the identity in the form of a unique serial number of an object or person wirelessly based on the radio waves. RFID can recognize target and obtain the related information automatically. RFID consists of reader, data accumulator and electronic tags. The reader works as a transceiver or an interrogator and emits electronic signals to the RFID tags by the radio waves [14]. By scanning the RFID tags, the data stored in the tags are read and retrieved, and then sent to the data accumulator. The electronic tags are used to store the related information [15]. In this paper, RFID is used to get the data collected by the above sensors.

\section{EXPERIMENT AND CONCLUSION}

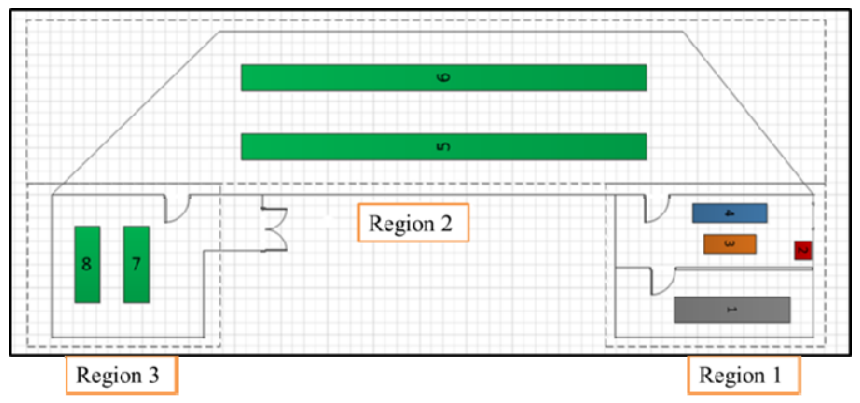

FIGURE I. THE LAYOUT OF OUR TELECOMMUNICATION ROOM

Figure 1 shows the layout of our telecommunication room. The telecommunication room is divided into three regions as shown in Figure 1. The specified sensors and the number of corresponding sensors used in our system are shown in Table 1. In the region 1, 7 temperature and humidity sensors, 10 Temperature sensors, 4 infrared lattices, 2 water leakage sensors and 1 smoke sensor are used in our system to realize the green telecommunication room.

After the text edit has been completed, the paper is ready for the template. Duplicate the template file by using the Save As command, and use the naming convention prescribed by your conference for the name of your paper. In this newly created file, highlight all of the contents and import your prepared text file. You are now ready to style your paper; use the scroll down window on the left of the MS Word Formatting toolbar.

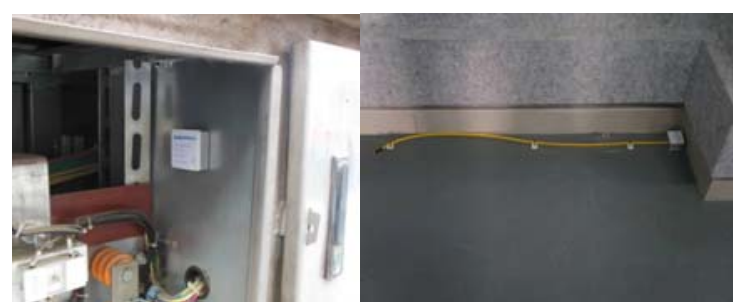

(a)Temperature and humidity sensor (b) Water leakage sensor

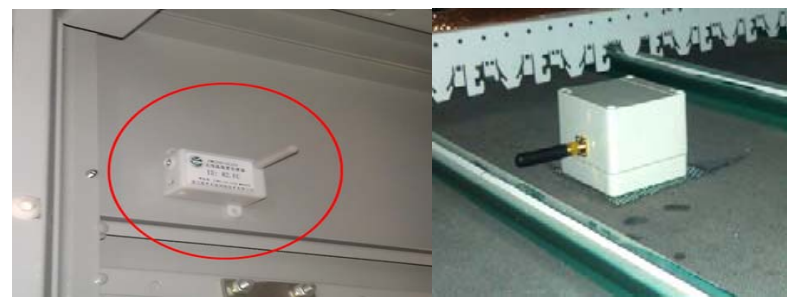

(c)Temperature sensor

(d) Infared lattice

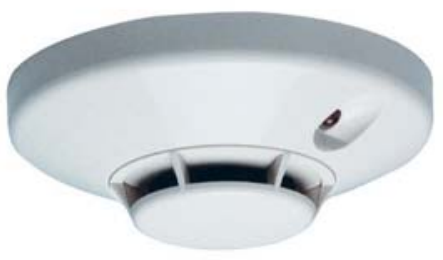

(e) Smoke sensor

FIGURE II. THE SENSORS USED IN OUR SYSTEM

Figure 2 provides an example of the sensors used on our system. Figure 2 (a) shows an example of the temperature and humidity sensor. Figure 2 (b) shows an example of the water leakage sensor. Figure 2 (c) shows an example of the temperature sensor. Figure 2 (d) provides an example of the infrared lattice, and Figure 2 (e) shows an example of the smoke sensor.

The data are collected by the sensors and transmitted to the data module by the Wireless data base station. Figure 2 shows an example of the wireless data base station used in our system. The data modules are attached with RFID tags. Via scanning the RFID tags by the RFID reader, data about the telecommunication can be obtained for analysis and realize the green telecommunication room.
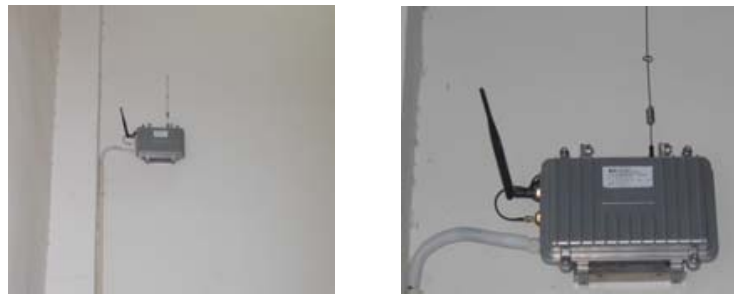

FIGURE III. WIRELESS DATA BASE STATION

\section{CONCLUSION}

In this paper, we proposed a system to realize the green telecommunication room based on the sensors and the RFID. The data are collect by the sensors and transmitted to the data accumulator by the base station. Afterwards, by scanning RFID tags, the data of the operation of the telecommunication can be obtained and fed back to the specific device or people to make corresponding changes. Further, the collected data can be used for analysis of the operation of the telecommunication, and make optimal scheme for reducing the cost and keeping the stable operation.

Our future work will focus on extending the application and optimizing our scheme. Swarm intelligence, such as biogeography-based optimization [16], particle swarm 
optimization [17], artificial bee colony [18], etc., will be tested to augment the performance

\section{REFERENCE:}

[1] Lee, J.S., J. Oh, J. Jun, et al., Wireless Hydrogen Smart Sensor Based on Pt/Graphene-Immobilized Radio-Frequency Identification Tag. Acs Nano, 2015. 9(8): p. 7783-7790.

[2] Zhou, X., S. Wang, W. Xu, et al., Detection of Pathological Brain in MRI Scanning Based on Wavelet-Entropy and Naive Bayes Classifier, in Bioinformatics and Biomedical Engineering, F. Ortuño and I. Rojas, Editors. 2015, Springer International Publishing: Granada, Spain. p. 201209.

[3] Pumpoung, T. and C. Phongcharoenpanich, Design of Wideband Tag Antenna for Ultra-High-Frequency Radio Frequency Identification System Using Modified T-Match and Meander-Line Techniques. Electromagnetics, 2015. 35(5): p. 340-354.

[4] Choi, J., J. Jeon, and Y. Kim, Cooling performance of a hybrid refrigeration system designed for telecommunication equipment rooms. Applied Thermal Engineering, 2007. 27(11-12): p. 2026-2032.

[5] Chen, J., The application of computer room power environment comprehensive monitoring system in Maritime Communications. Telecommunications Technology, 2012. 11: p. 225-228.

[6] Yang, S., Solution of computer room system's security. Intelligent building, 2005. 10: p. 30-32.

[7] Okundamiya, M.S., J.O. Emagbetere, and E.A. Ogujor, Design and control strategy for a hybrid green energy system for mobile telecommunication sites. Journal of Power Sources, 2014. 257: p. 335343 .

[8] Bu, Y., G. Lu, and Y. Huang, Design of Supervisory and Management System of Network Center Computer Room Devices. Modern Architecture Electric, 2010. 3: p. 5-7.
[9] Wang, S., H. Pan, C. Zhang, et al., RGB-D image-based detection of stairs, pedestrian crosswalks and traffic signs. Journal of Visual Communication and Image Representation, 2014. 25(2): p. 263-272.

[10] Zhang, Y., S. Wang, P. Phillips, et al., Binary PSO with mutation operator for feature selection using decision tree applied to spam detection. Knowledge-Based Systems, 2014. 64(0): p. 22-31.

[11] Choi, S.H., B. Yang, and H.H. Cheung, A mechanised 3D scanning method for item-level radio frequency identification of palletised products. Computers in Industry, 2015. 72: p. 36-46.

[12] Guo, D., Y. Zhang, Q. Xiang, et al., Improved Radio Frequency Identification Indoor Localization Method via Radial Basis Function Neural Network. Mathematical Problems in Engineering, 2014.

[13] Tsirmpas, C., A. Rompas, O. Fokou, et al., An indoor navigation system for visually impaired and elderly people based on Radio Frequency Identification (RFID). Information Sciences, 2015. 320: p. 288-305.

[14] Yang, G., Y. Zhang, J. Yang, et al., Automated classification of brain images using wavelet-energy and biogeography-based optimization. Multimedia Tools and Applications, 2015: p. 1-17.

[15] Hossain, M.A. and M. Quaddus, Radio frequency identification (RFID) adoption: A cross-sectional comparison of voluntary and mandatory contexts. Information Systems Frontiers, 2015. 17(5): p. 1057-1076.

[16] Wang, S., Y. Zhang, G. Ji, et al., Fruit Classification by WaveletEntropy and Feedforward Neural Network Trained by Fitness-Scaled Chaotic ABC and Biogeography-Based Optimization. Entropy, 2015. 17(8): p. 5711-5728.

[17] Zhang, Y., S. Wang, Z. Dong, et al., Pathological brain detection in magnetic resonance imaging scanning by wavelet entropy and hybridization of biogeography-based optimization and particle swarm optimization. Progress In Electromagnetics Research, 2015. 152: p. 4158.

[18] Wu, L. and S. Wang, Magnetic Resonance Brain Image Classification by an Improved Artificial Bee Colony Algorithm. Progress in Electromagnetics Research, 2011. 116: p. 65-79.

TABLE I. THE NUMBER OF THE CORRESPONDING SENSORS USED IN OUR SYSTEM

\begin{tabular}{|c|c|c|c|c|c|c|c|}
\hline Sensors & $\begin{array}{c}\text { Temperature and } \\
\text { humidity sensor }\end{array}$ & $\begin{array}{c}\text { Temperature } \\
\text { sensor }\end{array}$ & $\begin{array}{c}\text { Infrared } \\
\text { lattice }\end{array}$ & $\begin{array}{c}\text { Water leakage } \\
\text { sensor }\end{array}$ & $\begin{array}{c}\text { Smoke } \\
\text { sensor }\end{array}$ & $\begin{array}{c}\text { DC } \\
\text { monitor }\end{array}$ & $\begin{array}{c}\text { Battery collection } \\
\text { module }\end{array}$ \\
\hline Region 1 & 7 & 10 & 3 & 2 & 1 & 2 & 48 \\
\hline Region 2 & 32 & 51 & 11 & 8 & 10 & 2 & 0 \\
\hline Region 3 & 2 & 4 & 0 & 4 & 2 & 4 & 48 \\
\hline
\end{tabular}

